\title{
Fully 3D Printed Flexible, Conformal and Multi- directional Tactile Sensor with Integrated Biomimetic and Auxetic Structure
}

\section{Yuyang Wei}

The University of Manchester https://orcid.org/0000-0002-3200-8598

\section{Bingqian Li}

Jilin University

\section{Marco Domingos}

University of Manchester https://orcid.org/0000-0002-6693-790X

\section{Yiming Zhu}

The University of Manchester

\section{Lingyun Yan}

The University of Manchester https://orcid.org/0000-0002-9986-2182

Lei Ren ( $\nabla$ lei.ren@manchester.ac.uk)

University of Manchester

\section{Guowu Wei}

University of Salford https://orcid.org/0000-0003-2613-902X

\section{Article}

Keywords: tactile sensors, 3D printing, biomimetics

Posted Date: September 20th, 2021

DOl: https://doi.org/10.21203/rs.3.rs-877986/v1

License: (c) (i) This work is licensed under a Creative Commons Attribution 4.0 International License. Read Full License 
1 Fully 3D Printed Flexible, Conformal and Multi-directional Tactile

2 Sensor with Integrated Biomimetic and Auxetic Structure

3 Author Information

4 Affiliations

$5{ }^{1}$ School of Mechanical, Aerospace and Civil Engineering, The University of Manchester,

6 Manchester, UK

$7 \quad{ }^{2}$ Key Laboratory of Bionic Engineering, Ministry of Education, Jilin University, China

$8 \quad{ }^{3}$ The Institute of Mechanical Engineering, University of Salford, Manchester, UK

9 Yuyang Wei-1, Bingqian Li-2, Marco Domingos-1, Yiming Zhu-1, Lingyun Yan-1, Lei Ren*-1,2 and

10 Guowu Wei*-3

11 Contributions

12 YW designed, fabricated the sensor, performed the experiments, analyzed the data, and wrote the paper.

13 MD helped to revise the paper and give advice. BL, YZ and LY performed some experiments. LR and

14 GW supervised the whole research and analyzed the data.

15 Corresponding author

16 Lei Ren* and Guowu Wei*, Email: lei.ren@manchester.ac.uk or g.wei@salford.ac.uk

17

18

19

20

21

22

23 


\section{Abstract}

25 Tactile sensors are instrumental for developing the next generation of biologically inspired robotic

26 prostheses with tactile feedback. Despite significant advancements made in current sensing technology,

27 several limitations still exist including the reduced sensing sensitivity under high pressure, lack of

28 compliance of the planar sensor with working surfaces and the demand for sophisticated manufacturing

29 processes. In this study, we investigate the feasibility of using the 3D printing technology for the rapid

30 and simple fabrication of a new conformal tactile sensor with an improved linear sensing range. The

31 auxetic structure is integrated with a biomimetic inter-locked papilla feature which allows to detect multi-

32 directional stimuli. Using the proposed design, the linear sensing range is extended to $0.5 \mathrm{MPa}$ and

33 responsive to normal and shear forces with the sensitivities of $2.42 \mathrm{KPa}^{-1}$ and $2.20 \mathrm{~N}^{-1}$ respectively. The

34 proposed tactile sensor was printed on the fingertip of a prosthetic robotic hand to perform the

35 sensorimotor control, or on the proximal femur head and lumbar vertebra for monitoring the bone-onbone load. The results have shown promising application prospects of the proposed tactile sensor.

\section{Introduction}

Over the past decade, great attention has been dedicated to the development of wearable, flexible tactile

40 sensors or electronic skin for applications in prosthetics, robotics and healthcare monitoring devices ${ }^{1-11}$.

41 Independent of applications, tactile sensors should be able to detect multi-directional stimuli, and for that

42 purpose different strategies have been proposed including the use of advanced composite materials, multi-

43 layered biomimetic or hierarchical structures ${ }^{6,10-13}$. Complex manufacturing approaches such as molding,

44 photolithography and etching technologies are traditionally employed in the fabrication of high-

45 performance sensors ${ }^{14-17}$ and often require sophisticated methods for the preparation of the materials.

46 Most of the existing tactile sensors have a planar structure which does not allow for optimal fixation on

47 uneven working surfaces, thus limiting their range of applications. To exploit the full potential of sensing

48 technology and deliver the next generation of prosthetics, alternative strategies must be developed, 
enabling high performance tactile sensors with more complex structures to be rapidly manufactured at

50 feasible costs.

51 In an attempt to improve the sensitivity and linear sensing range of tactile sensors, different researchers

52 have reported the combination of highly conductive composite materials such as metallic particles, 53 nanowires ${ }^{6}$, carbon nanotubes $(\mathrm{CNT})^{18}$, graphene palate ${ }^{19}$ with an insulation matrix. Microspheres can

54 also be integrated with nanomaterials, forming hierarchical structures with enhanced piezoresistive 55 properties ${ }^{12,13,19-22}$. Carbon nanotubes (CNT)/polymer composites have also been widely exploited in the generation of dielectric layers due to their high axial conductivity. When compared to similar quantities of

57 carbon black or graphene, the addition of CNTs to polymer matrices can induce a substantiality higher 58 piezoresistive response with minimal effect on the mechanical properties of the composite ${ }^{23-25}$. Volatile 59 or water soluble micro-particles were also employed by researchers to generate sponges or porous 60 material ${ }^{8,19}$, increasing the self-contact area and linear sensing range. However, the process for producing 61 these multiscale and hierarchical materials is time-consuming and technically complicated ${ }^{20}$. The stability 62 and homogeneity of the material cannot be precisely controlled thus affecting the final structure and 63 performance of the sensor. In particular, the sensitivity and linear sensing range, can be improved through 64 the use of porous or hollow structures ${ }^{8,18,19}$, multiscale hierarchical structures $5,6,20$, biomimetic 65 interlocked structures ${ }^{11,22,26,27}$, mechanosensory hair or even crack like structures ${ }^{28,29}$. In a recent study, 66 Park et al. has reported the fabrication of a series of interlocked micro-dome arrays capable of amplifying 67 the signal response whilst differentiating between multiple stimuli directions ${ }^{10,11,20}$. With the same goal in 68 mind, other researchers have used multilayer or hierarchical hair-like interlocked geometries ${ }^{6,9}$ to push 69 the boundaries of sensitivity and obtain an extra low detection threshold of $0.6 \mathrm{~Pa}$. However, these 70 structural features need a sophisticated and time-consuming fabricating process. The enhanced 71 performance reported in these studies still does not translate into a wider use of sensing technology. One 72 of the reasons may be related to the complexity of the manufacturing processes and associated costs, still 73 prohibitive for many research groups and industries ${ }^{14-17}$. The use of advanced composite materials could 
74 be seen as an alternative to the above strategies, but the long production time and high-demanding 75 operating environments are limiting factors when considering their use at industrial scale. Another 76 important issue that remains unsolved is the generation of a strong, stable and durable interface between 77 the sensor and the working surface ${ }^{26,30}$.

78 In this paper, we propose a new approach based on the use of 3D printing technology and advanced 79 materials to fabricate a biomimetic tactile sensor, which can detect both normal and shear forces. The 6

80 by 6 flexible tactile sensor (Fig. 1a) can be printed directly onto any uneven surfaces, such as the fingertip 81 of a biomimetic hand for sensorimotor control, or the vertebra and femur for bone-on-bone load 82 monitoring. When compared with other tactile sensors made of similar piezoresistive material, the 83 integrated biomimetic inter-locked and auxetic structure (Fig. 1b) of the proposed sensor lead to improved 84 performance at different levels, namely: 1) higher sensitivity to strain variations; 2) larger linear sensing 85 range and sensitivity due to negative Poisson's ratio; 3) easy detection of stimuli direction. More than 86 simply overcoming the limitations of the current technology, this study aims to provide new tools towards 87 the design and fabrication of pressure sensors with user-defined auxetic features tailored for specific 88 applications.

89 Results

90 The structure design and 3D printing of the tactile sensor

91 The proposed tactile sensor array was fully 3D printed containing the upper and lower papilla-auxetic 92 dielectric layer sandwiched between two flexible electrode layers. Fig. 1a-e present the layout of the 93 flexible tactile sensor array consisting of 36 sensing elements $\left(2 \times 2 \mathrm{~mm}^{2}\right.$ for each sensing element). The 94 sensing area for each element is similar to the receptive filed of the slowly adapting type I (SAI) 95 mechanoreceptor 31 to ensure a close match between the sensing capability of our sensor and that of the 96 human subject. The biomimetic inter-lock structure was integrated to enhance the sensitivity and 97 differentiating the directions of the external stimuli. Our results show that piezoresistive sensing elements 98 (located at the position of each small papilla) placed on the side of the applied force experienced 
99 substantially larger pressure and reduction of resistance compared to those placed on the opposite side.

100 This can be associated with the geometric features of the lower large papilla and the anisotropic 101 deformation of the upper dielectric layer with small papilla. The direction of the shear force could be 102 intuitively differentiate based on each large papilla (Fig. 1f), surrounded by four small papillae above its 103 four corners. The two small papillae on the side of the shear force experienced larger current increments 104 than the other two on the opposite side. Therefore, the pressure mapping on the tactile sensor array, 105 originated by the anisotropic distribution of resistance around the lower papilla, provided the ability to 106 detect and differentiate between shear forces with different directions (four in total). On the other hand, 107 the single sensing element was not able to provide the bulk information on force direction.

108 A CNT-Silicon rubber composite was used as the printing material for fabricating this high109 performance tactile sensor. The multi-wall CNT was evenly distributed within the silicon matrix (Fig. 2a), 110 providing piezoresistive properties under external stimuli. The conductive micro-copper-silicone 111 composite was employed to print the electrode, more detailed information on its preparation can be found 112 under materials and methods section. Printability of developed composite materials was determined 113 through rheological measurements, in particular by measuring the variation of viscosity and shear stress 114 as a function of shear rate (see Supplementary Fig. 1 and 2). The viscosities of both composites were 115 decreasing under shear strain during amplitude sweep test which means these composites could be used as $1163 \mathrm{D}$ printing materials in terms of the rheology properties. 


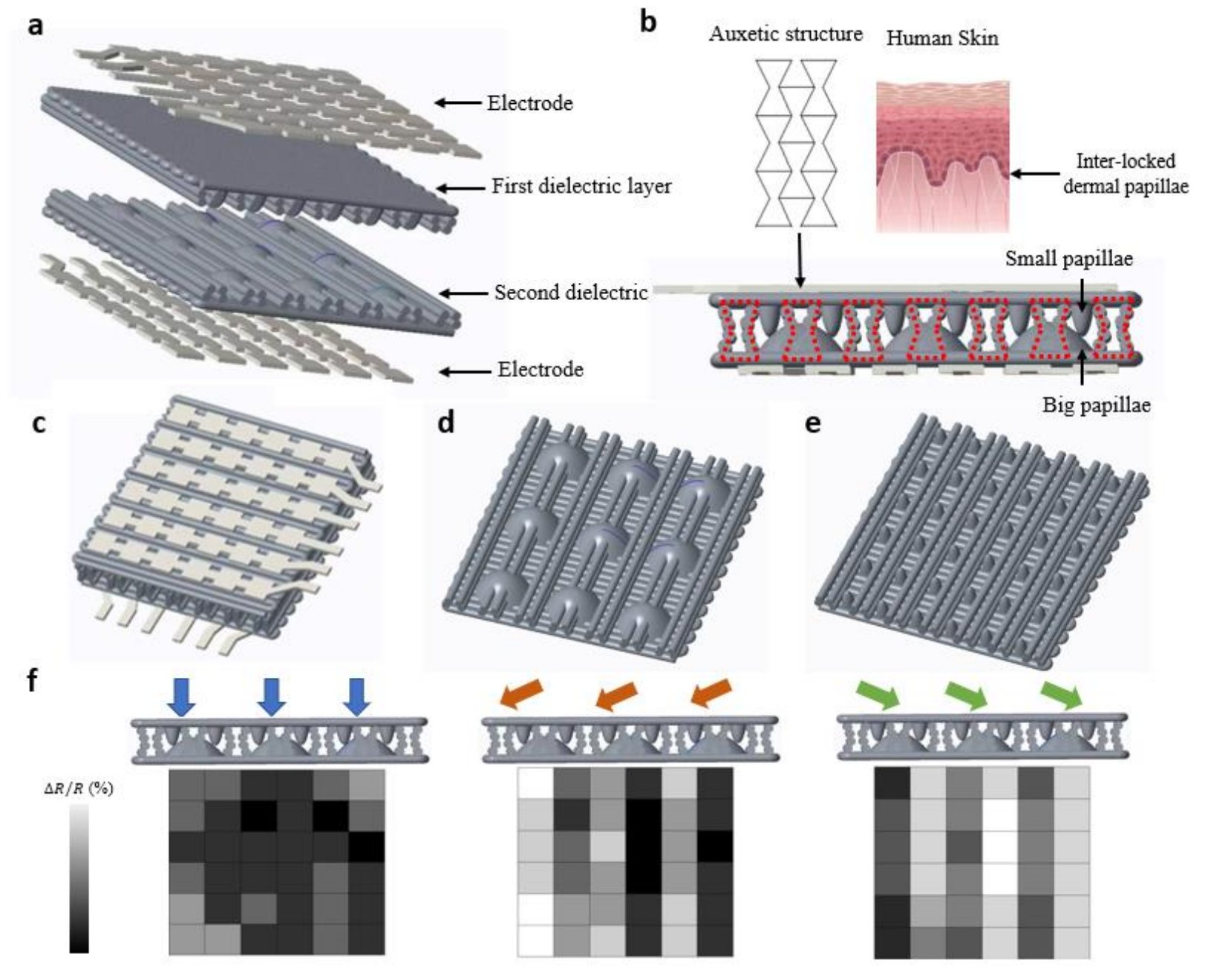

Fig.1 The structure and working principle of the tactile sensor. (a). A standard $6 \times 6$ tactile sensor array. Each sensing element corresponds to a small papilla of the inter-locked structure. (b). The integrated auxetic and biomimetic inter-locked structure of the sensor. The auxetic structure is highlighted in red dash line, the small and large papilla are located on upper and lower dielectric layer, respectively. (c) The 3D model of the sensor. The sensor is composed by a dielectric layer sandwiched between two layers of electrodes. (d) The lower dielectric layer of the sensor consists of nine large papilla structures and auxetic lines. (e). The upper layer of the sensor consists of 36 small papilla structures and auxetic lines. (f) The stimuli are applied from different directions (indicated by the arrows above the sensor) and the corresponding pressure distribution shown through a customized graphical user-interface (GUI).

In order to print the electrode and dielectric layer, the composite material was loaded into a syringe and transferred to the multi-material 3D printer developed in house (Fig. $2 \mathrm{~d}$ to g). Afterwards, the double

131 layered sensor, comprising an upper and lower papilla-auxetic structures and containing the electrodes,

132 was directly fabricated according to the $G$ codes developed manually based on the 3D models. The 133 moving speed ranging from 5 to $15 \mathrm{~mm} / \mathrm{s}$ (step increment $1 \mathrm{~mm} / \mathrm{s}$ ) under the pressure varied from 0.5 to 
1341.5 bar (step increment 0.1 bar) were used to carry out the trail line printing, resulting in totally 121 135 different combinations of printing parameters. The pressure of 0.7 bar with printing speed of $12 \mathrm{~mm} / \mathrm{s}$ was 136 found achieving a stable printing quality and appropriate extrusion width. The pressure of 1 bar was set 137 for dot-printing the papilla structure.

138 Firstly, the electrode was directly written with a 15-gauge needle mounted on the syringe loaded with 139 silver coated copper-silicone composite material. Another syringe with the CNT-silicone composite was 140 replaced and mounted with 22-gauge needle for line printing auxetic structure of the lower dielectric layer. 141 A 10-gauge needle was then changed for dot printing the large papilla structure on top of the lower layer. 142 Similar printing parameters and patterns were applied for printing the upper papilla-auxetic structure. 143 Finally, the upper electrode was printed on top of the sensor. The working surface for printing the sensor 144 was discretized with a fine mesh in commercial FE software Abaqus (Dassault Systèmes Simulia Corp., $145 \mathrm{UK})$. The coordinates of the nodes were transferred to $\mathrm{G}$ code for printing in order to obtain a good 146 compliance between the printed path and the target surface. The printing process of the sensor is shown in 147 Movie 1. 
a

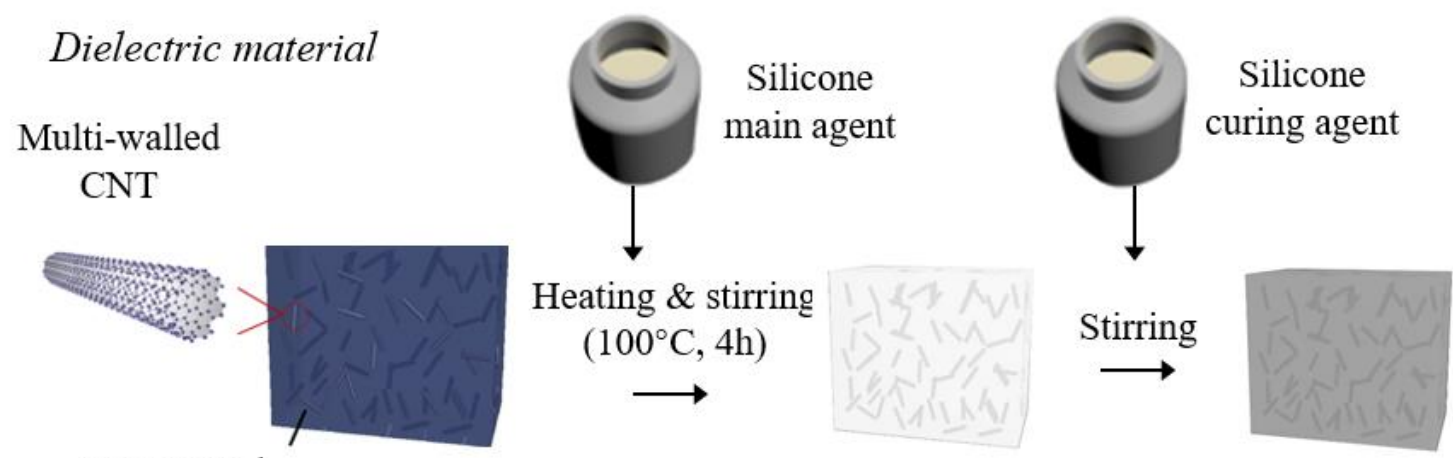

Isopropanol

CNT suspension

Printable CNT-silicone ink

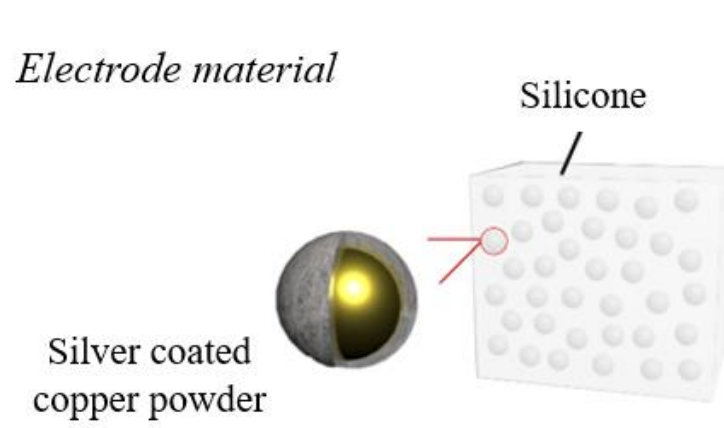

Silicone-copper composite
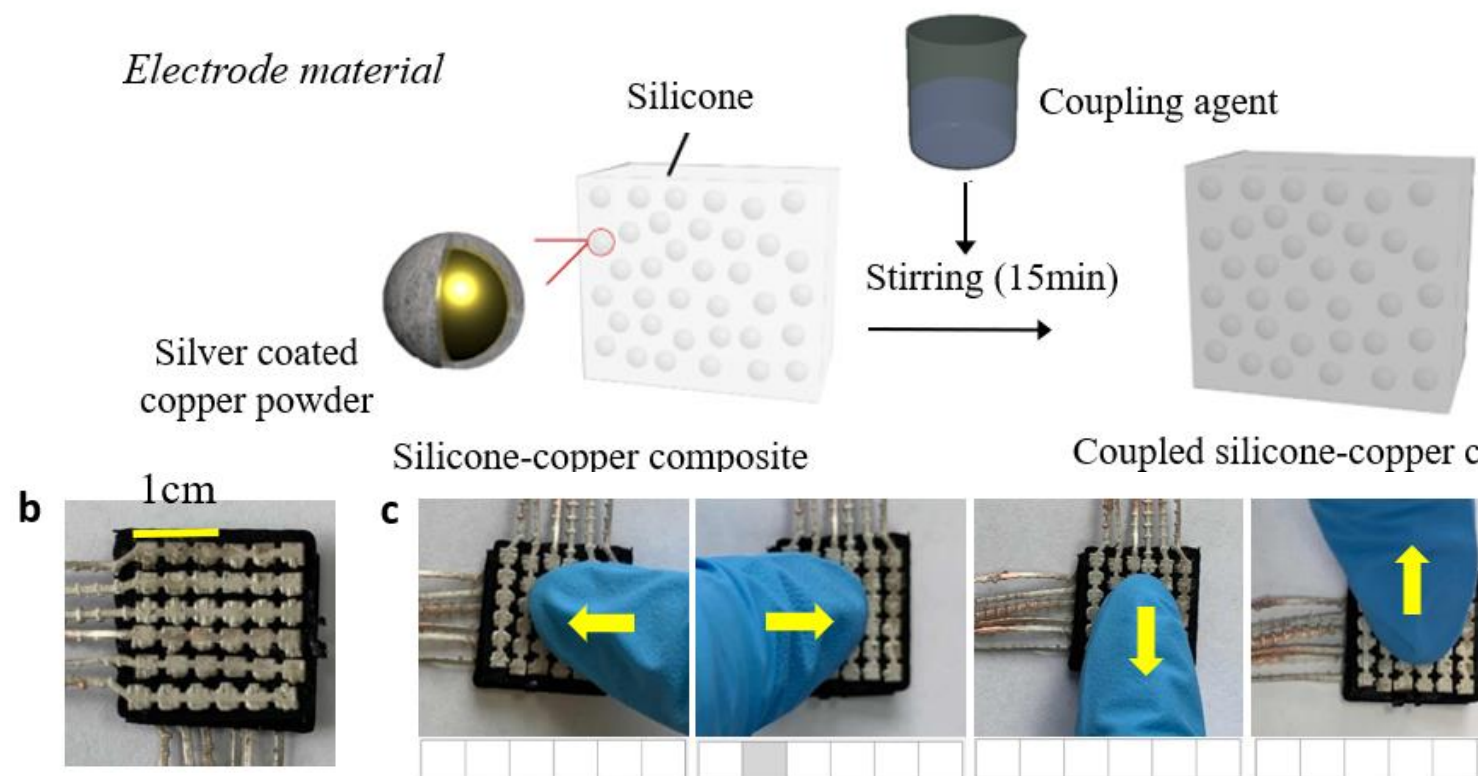

c
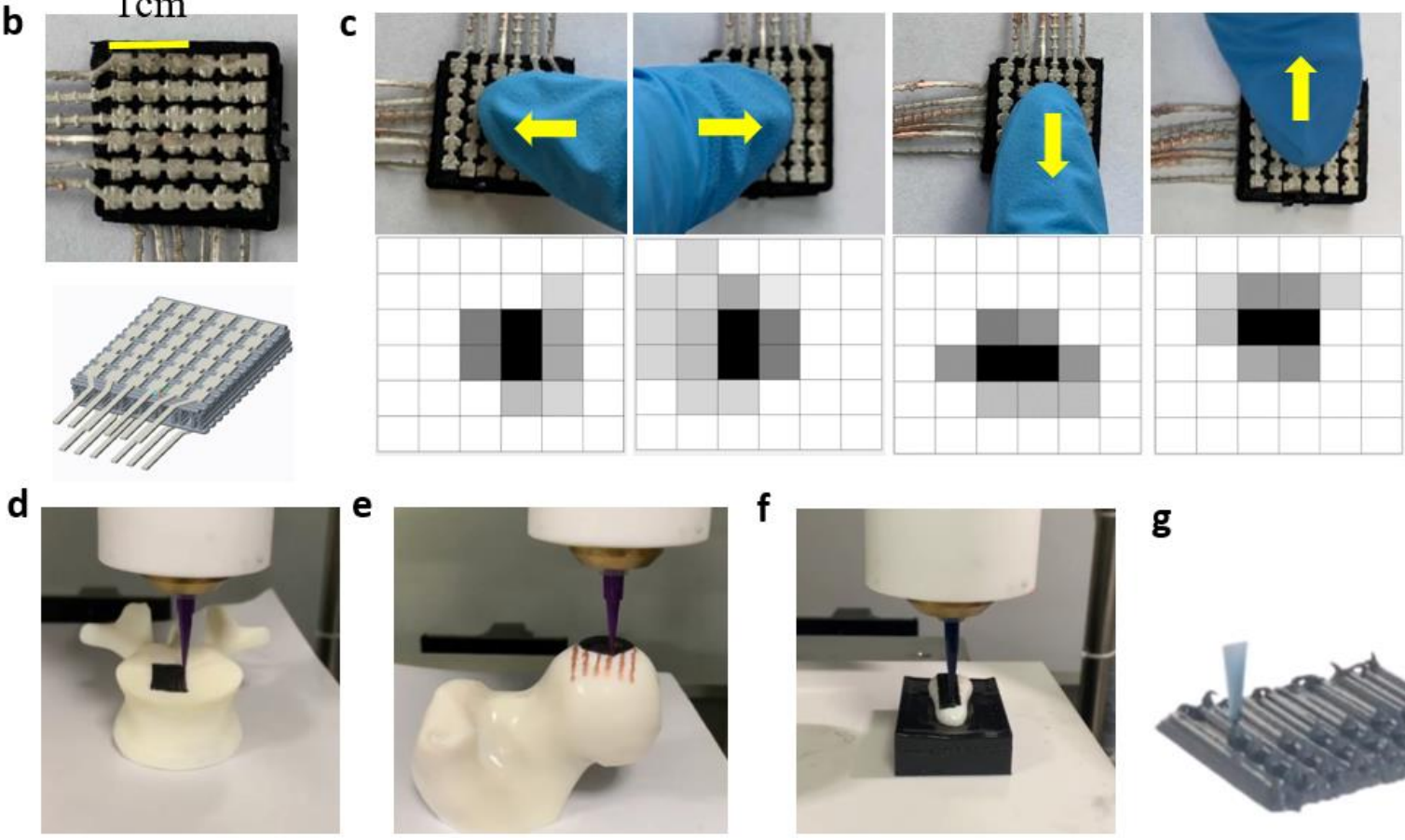

g

Fig.2. The material preparation and 3D printing of the sensor. (a) The material preparation of the silicone-CNT and silicone-copper composite (b) The physical prototype and 3D model of the printed sensor. (c) The fingertip contact force was applied on tactile sensor, the direction of the shear force was indicated by the arrows in yellow. The distribution of the contact pressure are shown through customized graphic user-interface, it can be seen that the sensing elements on the sides of the shear force experienced 
larger resistance variation. (D) The 3D printed sensor on a model of human vertebra. (E) The 3D printed sensor on the proximal femur head. (F) The 3D printed sensor on the distal index phalange. (G) The 'dot printing' for inter-locked papilla structure.

\section{Auxetic structure optimization of the tactile sensor}

Research has shown that the auxetic structure can provide negative Poisson's ratio and larger self-contact area 32. Therefore, the auxetic structure was applied to enlarge the linear sensing range and enhance the sensitivity. The thickness and re-entrant angle of the auxetic structure (Fig. 3a and b) was optimized to achieve the best nominal sensitivity and the predicted results were also validated against couple of experiments (Fig. 3b and c). The sensitivity (S) of the piezoresistive pressure sensor was defined as

$$
\mathrm{S}=(\Delta \mathrm{I} / \mathrm{I} 0) / \Delta \mathrm{P}
$$

in the linear regime, where I is the current flow over the dielectric layers and $\mathrm{P}$ is the applied pressure.

The nominal sensitivity ( $\mathrm{Sn}$ ) for optimizing the auxetic structure was defined as

$$
\mathrm{Sn}=(\Delta \mathrm{SED} / \mathrm{SED} 0) / \Delta \mathrm{P}
$$

Where SED stands for the strain energy density at the site of the sensing elements.

Research on piezoresistive characteristics of the elastic polymer-based sensor has shown that the sensitivity is dominated by the geometry of the structure and the stress/strain experienced within the composite 33-35. Due to the relatively small variations in the geometry of thin film-like flexible sensors, the change of strain energy density relative to the external pressure was considered as the main factor affecting the piezoresistive effect. Therefore, the variation of SED was employed instead of current for evaluating the nominal sensitivity. The structural parameters (Fig. 3a) to be optimized were re-entrant angle (from $45^{\circ}$ to $90^{\circ}$ with the increment of $1^{\circ}$ ) and $\mathrm{H} / \mathrm{L}$ (Fig. 2b) ratio (H/L=2.7, 2.0, 1.61 .31 .0 ). Totally 230 trails of simulation were performed in commercial FE software Abaqus. The sensor with reentrant angle of $65^{\circ}$ and the $\mathrm{H} / \mathrm{L}$ ratio of 1.60 achieved the largest nominal sensitivity according to the simulation results. Meanwhile, twenty tactile sensors with the re-entrant angle of $45^{\circ}, 60^{\circ}, 75^{\circ}, 90^{\circ}$ and five $\mathrm{H} / \mathrm{L}$ ratios were fabricated to validate the FE simulation results for structure optimization. Fig. 2a shows the results of the optimization and validation. The transparent mesh stands for the real sensor 
181 characterization results while the solid mesh represents the simulation results. Both the experiment and

182 simulation results suggested that the sensitivity and linear sensing range were significantly enhanced by

183 the integrated inter-locked and auxetic structure. The simulation results also reveal that different re-

184 entrant angles and H/L ratios of the auxetic features can significantly affect the sensitivity of the tactile

185 sensor. The simulation output of compression tests for the sensors with different auxetic features are 186 available in Movie. 2.

187

188
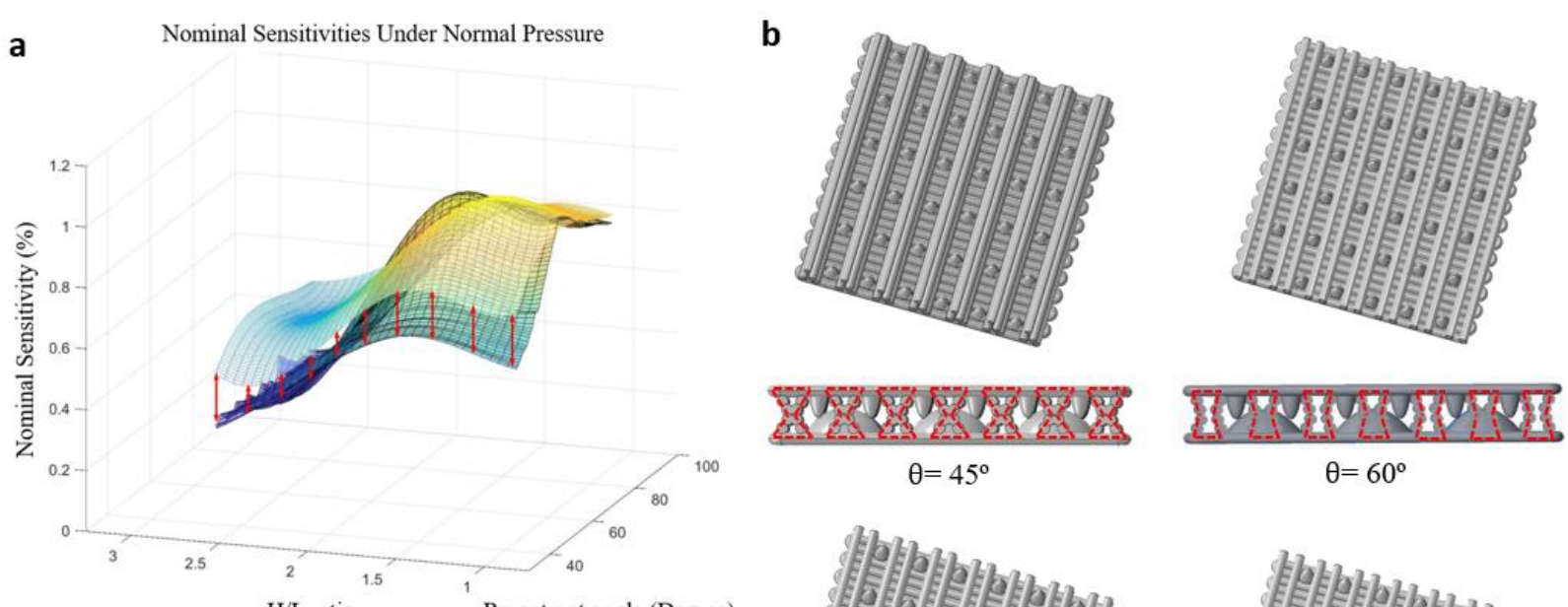

$\theta=45^{\circ}$

$\theta=60^{\circ}$
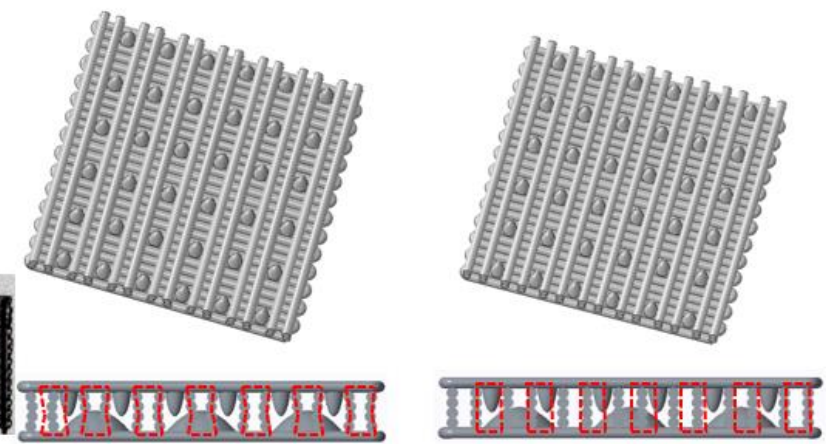

$\theta=45^{\circ}$

$\theta=60^{\circ}$

$\theta=90^{\circ}$

$\theta=90^{\circ}$

d

$\mathrm{H} / \mathrm{L}=1.6, \theta=45^{\circ}$
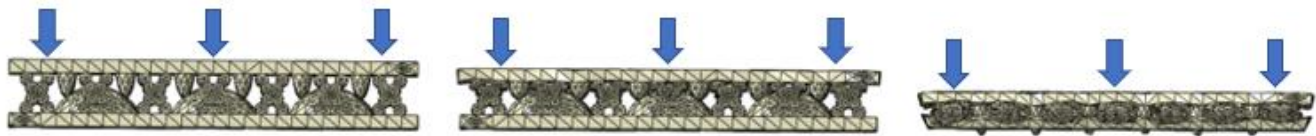

$\mathrm{H} / \mathrm{L}=1.6, \theta=60^{\circ}$
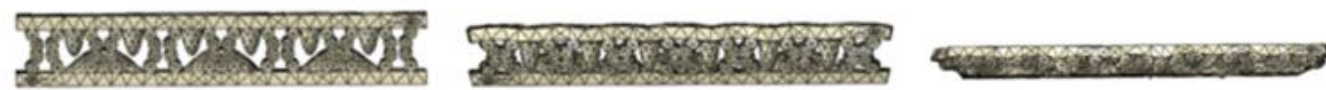

$\mathrm{H} / \mathrm{L}=1.6, \theta=75^{\circ}$
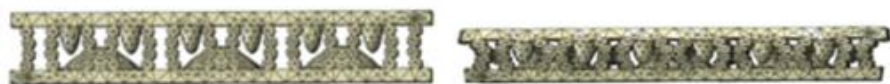
Fig. 3. The optimization results of the sensor and the prototypes with different structures. (a) The optimization results for the auxetic structure. The largest nominal sensitivity was achieved under the reentrant angle of $65^{\circ}$ and $\mathrm{H} / \mathrm{L}$ ratio of 1.6. The sensor with different auxetic structures were also fabricated to validate the simulation results. The tactile sensors with re-entrant angle of $45^{\circ}, 60^{\circ}, 75^{\circ}, 90^{\circ}$ and $\mathrm{H} / \mathrm{L}$ ratio of $1.06,1.3,1.6,2.0,2.7$, totally 20 samples with different geometric parameters were fabricated. Their sensitivities were measured and compared with the predicted nominal sensitivity derived based on the simulation results. The difference between simulation and experiment results were indicated in red arrows. The nominal and the measured sensitivities agreed well with each other. (b) The 3D model of the sensors with four different re-entrant angles modelled for structure optimization. The auxetic structures with different re-entrant angles are highlighted in red dash lines. (c) The fabricated prototype of the auxetic structure of the lower dielectric layer. The re-entrant angle of $45^{\circ}, 60^{\circ}, 75^{\circ}, 90^{\circ}$ were presented. (d) The simulation results of the compression experiments for the sensors with different auxetic structures. The sensors were shown in three stages: before during and after compression. The simulation results of the sensors with re-entrant angle of $60^{\circ}$ and different $\mathrm{H} / \mathrm{L}$ ratios are presented. The sensors before, during and after the compression are shown in supplementary Fig.3.

\section{Characterization of the sensor}

207 Fig.4 illustrates the characterization of this high-performance tactile sensor including the sensitivity (Fig. 4a, b), linear sensing range (Fig4. a, b), the lower detection limits and the response time (Fig. 4c). The sensitivity of the sensor with three different structures were compared in Fig.4d and 4f. The sensitivity

211 of the planar sensor was below $1 \mathrm{KPa}^{-1}$ while was significantly increased to $2.42 \mathrm{KPa}^{-1}$ by integrating

212 the interlocked papillae and optimized auxetic structure. Meanwhile the linear sensing range also

213 increased from 0.1 MPa to 0.5 $\mathrm{MPa}$, which is one order of magnitude higher compared with most of the

214 published high-performance sensors 1-3,11,18. The inter-locked features not only provide the sensor with

215 the ability of differentiating directions of the shear force but also improve its sensitivity. Importantly, the

216 integration of the auxetic structure allowed for enhanced sensitivity and extended the linear sensing range.

217 The tactile sensor was mounted onto a customized horizontal testing platform with a push-pull

218 dynamometer to generate the shear force (from 0 to $5 \mathrm{~N}$ ) while a universal testing machine was used to

219 provide the normal compression force. The shear sensitivity was quantified under the normal contact

220 pressures ranging from $0.2 \mathrm{KPa}$ to $10 \mathrm{KPa}$, the maximum shear sensitivity of $2.26 N^{-1}$ was found under the

221 normal pressure of $0.2 \mathrm{KPa}$ and reduced with the increased compression force. The shear sensitivity was

222 maintained at approximately $1.5 N^{-1}$ under the compression pressure ranging from 0.4 to $0.8 \mathrm{KPa}$ and 
223 was reduced to $0.83 N^{-1}$ when the normal pressure was above $1 \mathrm{KPa}$. It can be found from the Fig. $4 \mathrm{c}$ that

224 the shear sensitivity was significantly dominated by the inter-locked and auxetic structure of the sensor.

225 Only $0.37 N^{-1}$ of the sensitivity was achieved by the planar dielectric layer while it increased to $2.26 N^{-1}$

226 after integrating the biomimetic and auxetic features. The lower detection limit was found to be around

$22710 \mathrm{~Pa}$ as is presented in Fig. 4d, the response time is approximately $35 \mathrm{~ms}$ recorded by the multimeter. The

228 standard 36 sensing element tactile sensor was tested under repeated pressing and releasing cycles in a

229 wide range of compressive deformation. The stable signal response was achieved after 2000 loading-

230 unloading cycles.

231

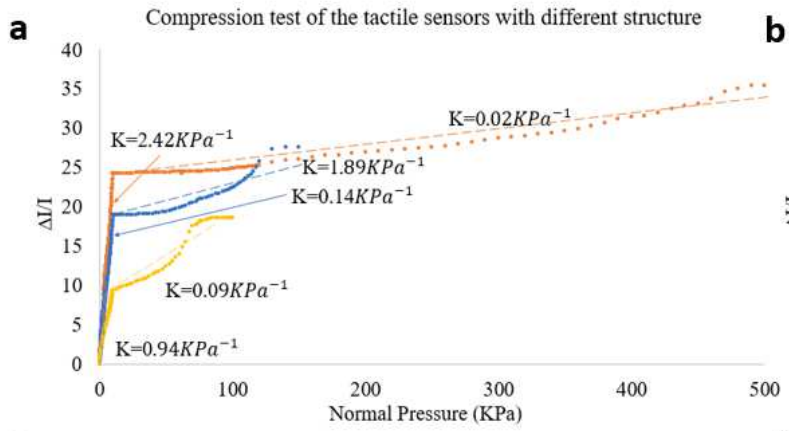

C

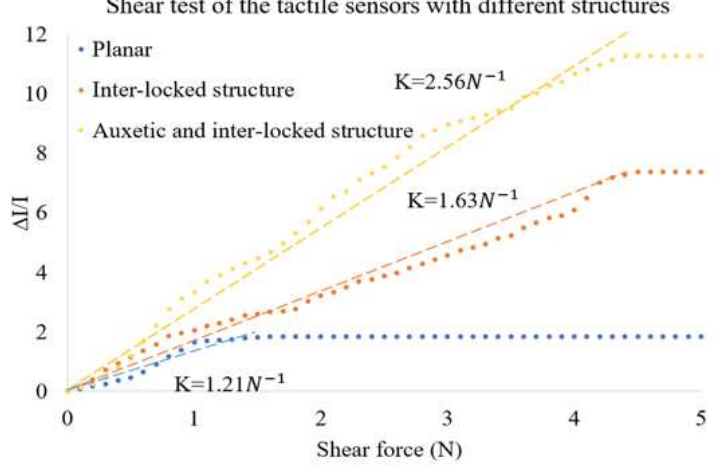

d

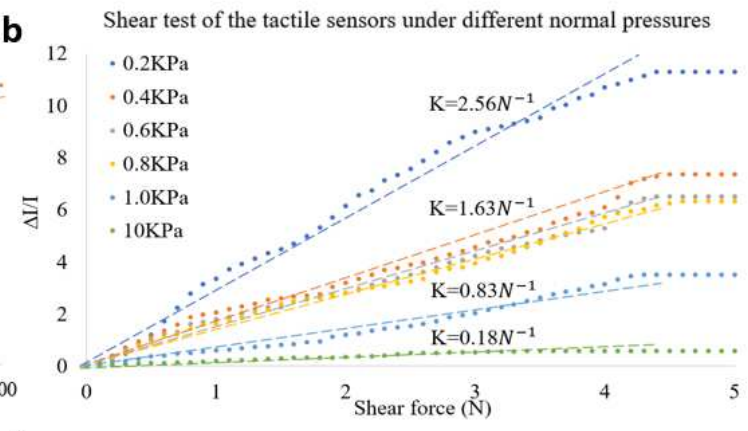
10000

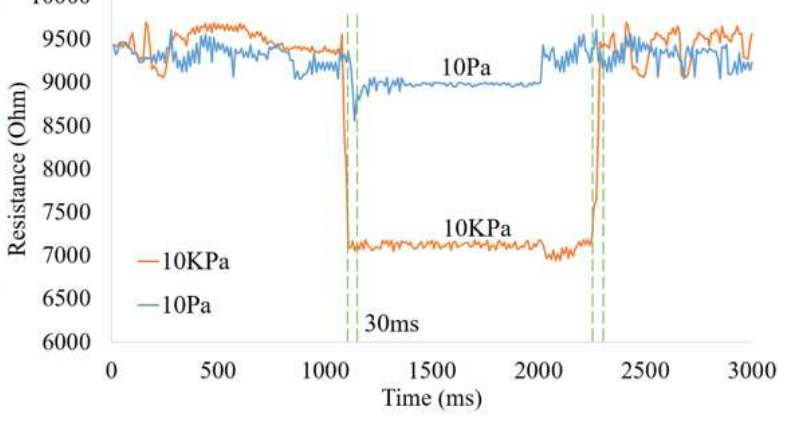




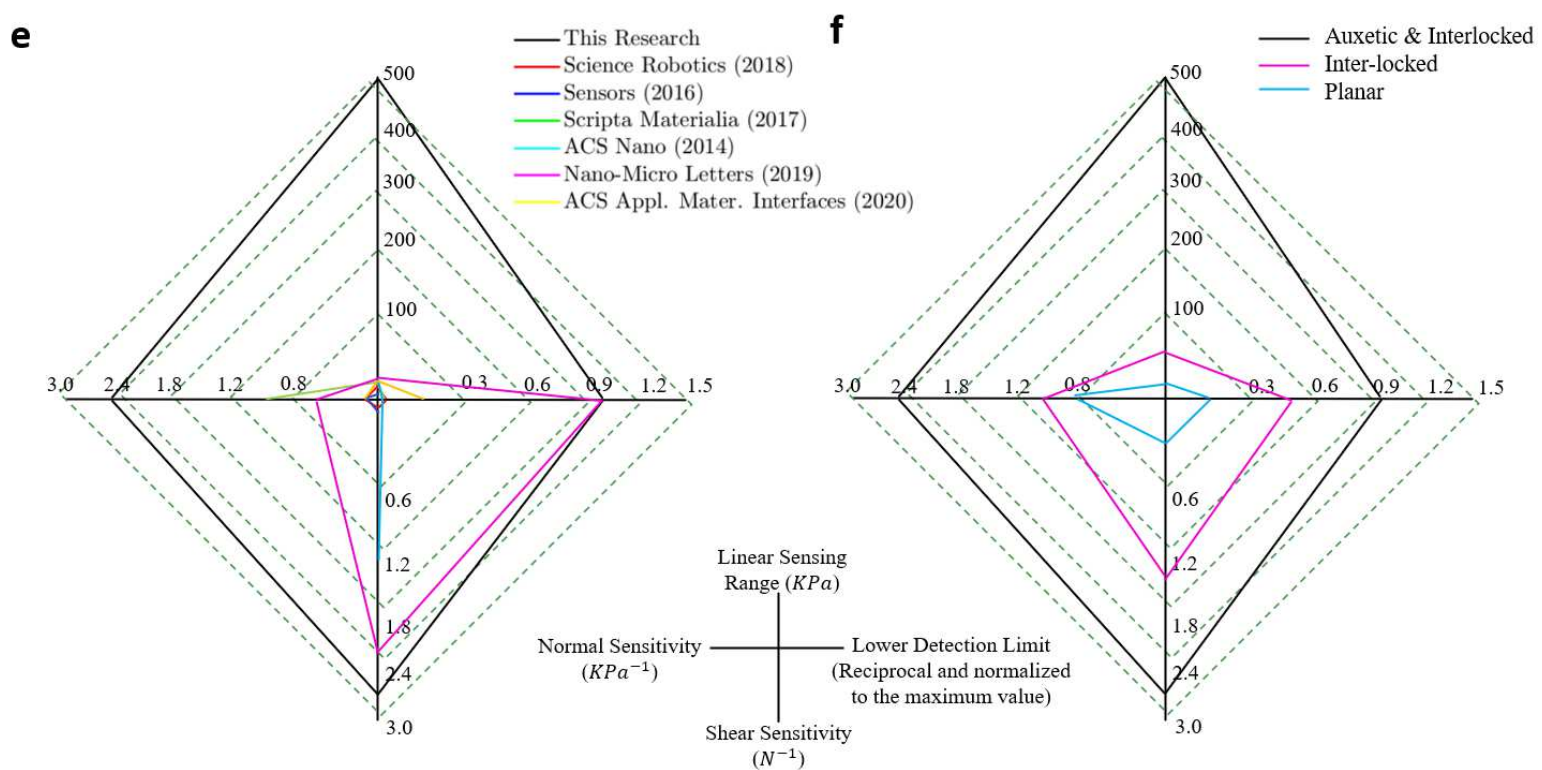

Fig. 4. The characterization and performance of the tactile sensor compared with other published sensors. (a). The sensitivity of the sensors with different structures including planar sensor, the sensor with the inter-locked structure and the sensor with both inter-locked and auxetic structure. The sensitivity was summarized within and beyond the 'gentle touch pressure, 10KPa'. (b) The shear sensitivity of the sensor under different normal pressures varied from 0.2 to $10 \mathrm{KPa}$. (c) The signal response of the sensor under the lowest detection limit 10Pa and gentle touch pressure 10KPa. (d) The shear sensitivity of the sensor with three different structures. (e). The comparisons of sensing performance between the published sensors and the tactile sensor developed in this study. The lower detection limitations were reciprocal and then normalized to the maximum value. Therefore, a larger index of the lower detection limit in the diagram means a better sensor performance. (f). The comparisons of sensing performances among the planar sensor, the sensor only adopted interlocked features and the one with the integrated inter-locked and auxetic structure.

\section{Monitoring magnitude and direction of the bone-on-bone load through the senor}

This fully 3D printed tactile sensor can be directly written onto uneven working surfaces such as the human vertebra and the proximal femoral head to monitor bone-on-bone load. To demonstrate the ability of the sensor array to detect both magnitude and direction of the contact force, the sensor was directly written onto the plateau of the human vertebra and used to monitor the intervertebral compression force and torque. The L2 and L3 vertebra together with the inter-vertebra disc (Fig. 5a to 5c) were 3D printed using UV curable resin as the working surface of the sensor. The model of the L3 vertebra was discretized in Abaqus to extract the coordinates of the working surface and defining a conformal printing path of the sensor. The printed pressure sensor was wired with a customized electric circuit for collecting the analog 
output from the $6 \times 6$ sensing elements and convert them into digital signals thus enabling visualizing on

257 GUI interfaces (Fig. 5d). Motions of the spine including lateral bending, axial rotation and

258 flexion/extension were performed under different compression forces ranging from 25 to $75 \mathrm{~N}$ with grey

259 color representing the output current and the magnitude of the pressure acting on each tactile element.

260 The distributions of these contact patterns indicate the direction and magnitude of the forces. It can be

261 seen from the customized GUI that the sensor performed well under the external stimuli including torque,

262 normal and shears forces, enabling visualization of the complex contact between intervertebral disc and

263 vertebra.

264 Due to the large linear sensing range, this sensor was also printed onto the proximal femoral bone to 265 monitor the large bone-on-bone load of the hip joint (Fig. 5e). A conformal printing path around the 266 femoral head was also defined to print the sensor. The proximal femoral head and part of the pelvis were 267 printed to perform the kinematics of the hip joint during the swing of the lower lime. The GUI shows a 268 reasonable pressure distribution during the whole swing phase confirming the ability of the pressure 269 sensor to detect multi directional loads (Fig. 5f). Also, the compression test of the sensor shown in Fig. 1f 270 and Fig. 2c present the capacity of this sensor to discriminate the directions of the stimuli based on the 271 biomimetic inter-locked structure. Therefore, the experimental results reported above suggest that this 3D 272 printed sensor can be rapidly fabricated onto geometrically complex surfaces such as the vertebra or 273 femoral head and used for monitoring the magnitude and direction of the external load. The printed 274 sensors working on hip joint and between the lumber vertebrae are presented in Movie. 3. 

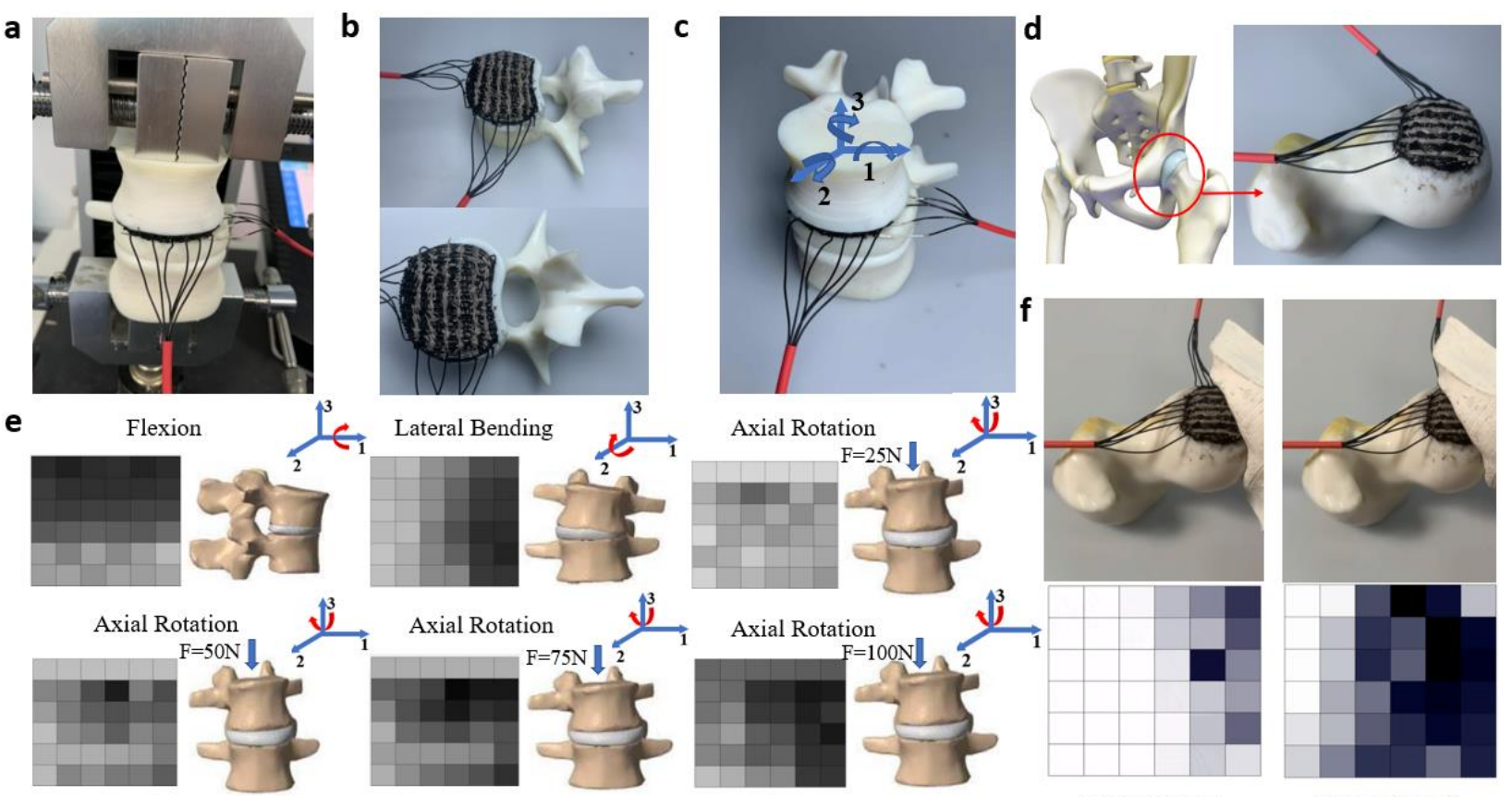

Fig. 5. The applications of the sensor on monitoring the bone-on-bone load. (a) The sensor was

278

278

279

280

281

282

283

284

285

286

287

288

289

290

291

292

293

294

295

printed onto the vertebra plateau to monitoring normal compression force. (b)(c) The printed sensor onto the vertebra plateau and the rotation around three axes corresponding to flexion/extension (axis 1), lateral bending (axis 2) and axial rotation (axis 3). (d) The printed sensor on the distal femur bone of the human hip joint. (e) The signal distribution of the sensor under different motions of the spine vertebra including flexion, lateral abending and axial rotation. The axial rotation was combined with different compression force of $25,50,75,100 \mathrm{~N}$ to show the sensor performance under different combinations of loading conditions. (f) The signal distribution of the sensor during the swing of hip joint.

\section{Sensorimotor control of the biomimetic hand through the tactile sensor}

The 6 by 5 tactile sensor array with approximate dimensions of $15 \times 12 \mathrm{~mm} 2$ was fully 3D printed onto the distal phalange of the biomimetic hand. The tendon-driven biomimetic hand employed in this study was reconstructed from a male subject, including the intact bone skeleton, tendon, interphalangeal ligaments and skin. Five motors were used to pull the tendon and achieved human like kinematics and grasping quality. The distal phalange of the index was disassembled from the biomimetic hand to print the sensor on it. A customized electric circuit was constructed to collect the analog output from the tactile sensing elements. A Python based controlling algorithm was developed to convert sensor analog output to digital signals as feedback for controlling the motors and producing contraction of the tendon. Detailed information about the hardware and controlling are illustrated in 'Materials and Methods' section. 
A plastic sphere was grasped by the biomimetic hand while a $0.75 \mathrm{~N}$ pulling force was randomly applied to provide the shear force. The tactile sensor was able to perceive the shear or slipping force on the fingertip and form a sensorimotor closed loop control to maintain the stable grasp. Fig. 6a and Movie. 4 show the reaction control of the biomimetic hand with the tactile feedback. Taken together, these results 300 clearly show the potential of the high-performance tactile sensor to monitor magnitude and direction of 301 stimuli, whilst providing feedback control of robotics by fast direct writing onto the target surfaces.

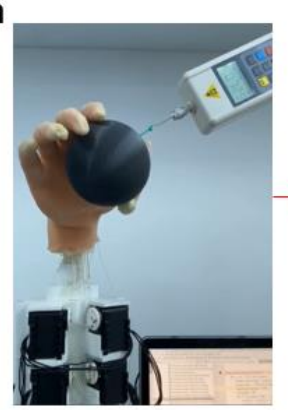

b

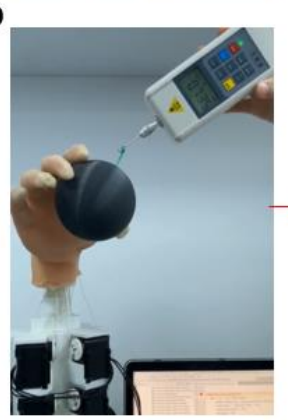

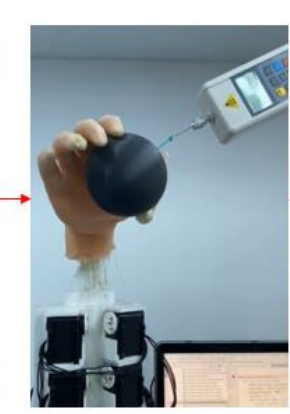
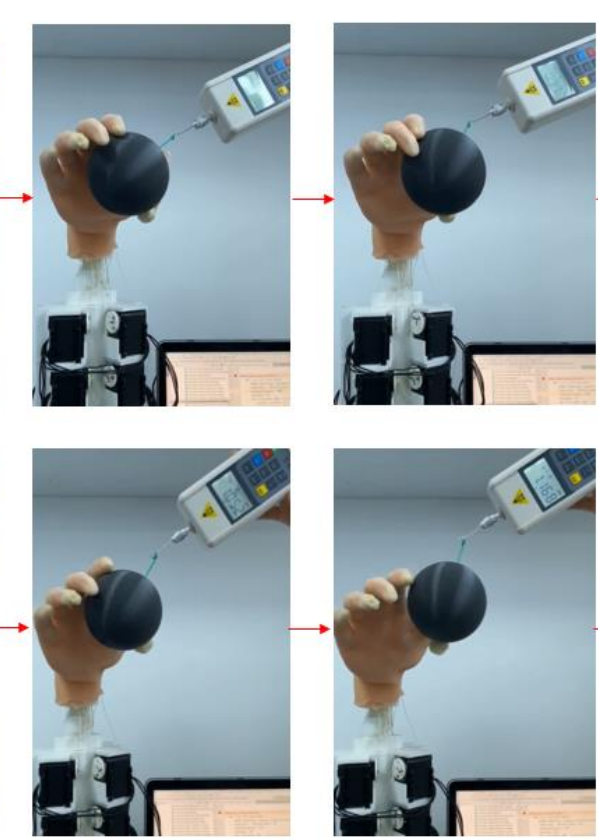

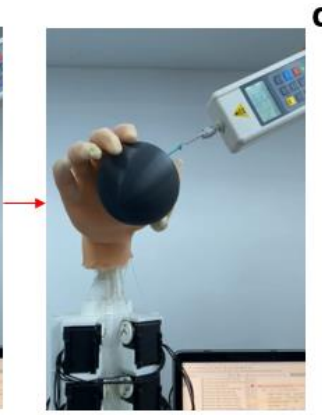

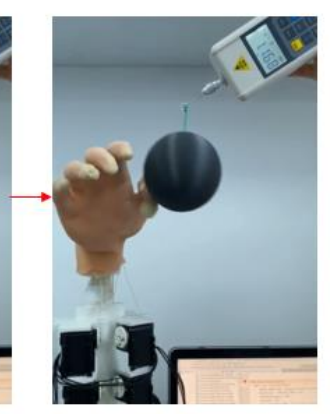
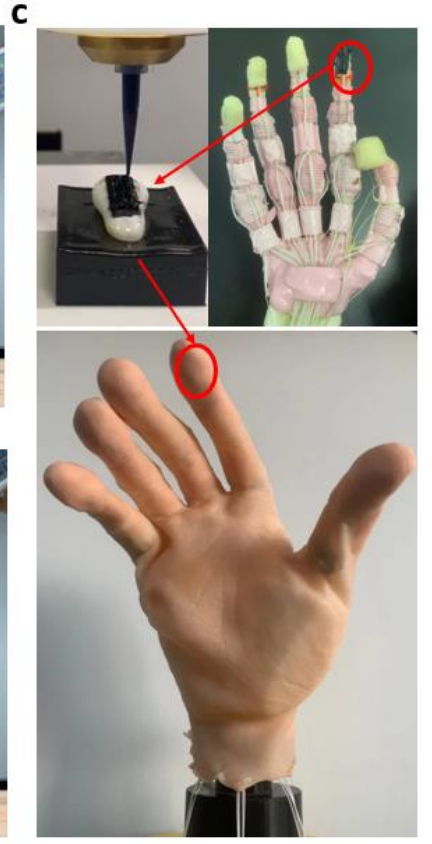

303

304

305

306

307

308

Fig.6. The closed-loop control of a biomimetic hand with tactile sensor printed on the index fingertip. (a) A plastic ball with diameter of $70 \mathrm{~mm}$ was grasped by the biomimetic hand, approximately $0.75 \mathrm{~N}$ pulling force was applied onto the ball. The biomimetic hand with tactile feedback from the sensor can sense the slip of the ball and grasp firmly. (b) The biomimetic hand without tactile feedback cannot produce a stable grasp. (c) The tactile sensor (indicated in red circle) was printed directly onto the distal phalange of index finger and then covered by the artificial skin as a biomimetic hand.

\section{Discussion}

Many tactile sensors were developed over the past decade while some of them were based on sophisticated fabricating process such as molding 7,13,18,37,38, photolithography, etching 14-17 which need high-demanding operating environment. Others employed the advanced composite material 6,9,13 resulted in a sophisticated process of material preparation. Also, the compliance between the tactile sensor 
and its working surface was rarely considered, the tactile or pressure sensors were supposed to be used on

316 arbitrary surfaces in many cases 39,40. A high-performance and easily fabricated tactile sensor compliant

317 with uneven surface is presented in this study. This 3D printed tactile sensor is able to detect the normal

318 and shear forces with sensitivities of $2.42 \mathrm{KPa}^{-1}$ and $2.26 \mathrm{~N}^{-1}$ which is in line with most of the existing

319 high-performance tactile sensors $1-3,11,18$. The tactile sensor proposed in this study was designed to

320 work on complex surface geometries, ensuring enhanced sensing capacity to detect direction and

321 magnitude of the stimuli, whilst being rapidly fabricated at feasible cost. The performance of our tactile

322 sensor including normal, shear sensitivity, linear sensing range and lower detection limit were compared

323 with some published sensors fabricated with similar composite material 2,3,11,18,20. As presented in Fig.

$3245 \mathrm{a}$, the linear sensing range of our sensor is at least one order of magnitude superior to the sensors in the

325 literature. The normal sensitivity is also larger than the published sensors, whilst the shear sensitivity and

326 lower detection limit of this tactile sensor are in line with others.

327 The inter-locked structure was employed to mimic the biomechanical characteristics of the human skin,

328 making the structure more sensitive to the variations of strain components while enabling the sensor to

329 detect the direction of the stimuli. Meanwhile, the auxetic structure is optimized and validated against the

330 experimental results. The re-entrant angle and thickness of the sensor are the main geometrical factors

331 dominating the negative Poisson's ratio effect of the auxetic structure. These two factors were optimized

332 with the nominal sensor sensitivity and the simulation results suggested that the re-entrant angle of $65^{\circ}$

333 and the $\mathrm{H} / \mathrm{L}$ ratio of 1.60 can provide the best piezoresistive effect. The self-contact area was significantly

334 increased by integrating the auxetic structure, leading to a wide linear sensing range. Simulation results

335 also suggested that the negative Poisson's ratio enabled a large self-contact area which was linearly

336 increased with the applied forces in the sensor with biomimetic and meta features. The sensing range

337 were significantly increased from 0.1 MPa to 0.5 MPa. Since the piezoresistive characteristics of the

338 elastic polymer-based sensor was dominated by the stress/strain related parameters during external stimuli,

339 the higher sensitivity might be caused by the sensitive SED variation to the external stimuli according to 
340 our simulation results. The normal sensitivity was enhanced from $0.94 \mathrm{KPa}^{-1}$ to $2.42 \mathrm{KPa}^{-1}$ with the

341 interlock and auxetic features, making the structure to be more responsive to the variation of normal

342 pressure, then leading to a better piezoresistive performance. The shear sensitivity was doubled (from

$3431.58 N^{-1}$ only with inter-lock features to $2.26 N^{-1}$ ) with the optimized authentic structure whilst the

344 lowest detection limit can reach to $10 \mathrm{~Pa}$ which is comparable with most of the published high-

345 performance tactile sensors 2,3,11,18.

346 The sensor in this study has shown that a better performance can be achieved by integrating the auxetic

347 or other Meta structure. The application of the optimized auxetic structure may provide new insights

348 towards the designing of tactile sensor in the future. The tactile sensor can be fully printed directly and

349 efficiently (rapid prototyped) onto any uneven surfaces such as the human phalange, proximal femur

350 bones and the plateau of lumber vertebra perfectly compliant with the attached surface. Fast and simple

351 fabrication process and the compatibility with arbitrary surfaces are critical for the future application of

352 tactile sensor.

353 In summary, a fully 3D printed flexible tactile sensor based on biomimetic inter-lock and auxetic 354 structure of CNT-Silicone rubber piezoresistive was developed for sensing the arbitrary contact force. The 355 3D direct writing technique was applied to print the CNT-Silicone and silver-coated powder-silicone 356 composite directly onto the working surfaces, fabricating the tactile sensors efficiently and economically.

357 Similar performance to most of the published high-performance tactile sensors was achieved 2,3,11,18.

358 The integrated biomimetic and auxetic structure dominate the high sensitivity and large linear sensing 359 range of the sensor. The auxetic structure proposed in this study can be effectively used to in the 360 structural design of pressure sensors due to its unique mechanical properties. The sensors were rapidly 361 fabricated onto its working surfaces like distal phalangeal bone, human vertebra and distal femur bone to 362 monitor the sophisticated biomechanical contact or providing sensorimotor control for robotic hand. With 363 the 3D printing technique and a simple material preparation process, the tactile sensor arrays can be 
manufactured efficiently and applied to working surfaces but with a better piezoresistive performance, durability than other sensors.

\section{Methods}

368 Material preparation for 3D printing

The high purity (95 wt\%) Multi-Walled CNT (XFM25, XFNano, China) was dispersed uniformly in isopropanol by ultrasonic dispersion at $26^{\circ} \mathrm{C}$ for $6 \mathrm{~h}$ to obtain a good CNT suspension (Fig. 2a). The silicone main agent (Polycraft GP3481-F Silicone rubber, MB Fibreglass, UK) was added into the CNTisopropanol suspension and was heated to $100^{\circ} \mathrm{C}$ under magnetic stirring until the isopropanol was completely evaporated. After cooling down to the room temperature, the silicone curing agent was added to the composite under the weight ratio of 10:1. Our research showed that the conductivity of the CNTsilicone composite can meet the requirement of the tactile sensor with $2 \mathrm{wt} \%$ of Multi-Walled CNT (Supplementary Fig. 4). Silver coated copper-powder-silicone composite (Fig. 3a) was used to print the electrode of the sensor. It was prepared by mixing the silver coated copper palate $(48 \mu \mathrm{m})$ with silicone rubber with the weight ratio of 1:3.5. The coupling agent (KH550) was then added under the weight ratio of 1:100 and mechanically mixed with the composite to improve the conductivity.

\section{Testing platform for sensor characterizations}

382 In order to take full advantage of the piezoresistive and mechanical properties of the interlocked-auxetic

383 features, the standard sensor with the sensing area of $2 \times 2 \mathrm{~cm}^{2}$ consisting of 16 sensing elements was

384 fabricated for characterization (Fig. 4). The sensor prototype was powered by 5V DC supply and 385 connected into a customized electronic circuit. The output current was measured and collected by the 386 multimeter (Keysight 34465A, Keysight Ltd., HK) at the frequency of 500Hz. The normal pressure 387 applied onto the sensor was controlled by the universal testing machine (WH-5000, Weiheng Co., China) 388 (above 10KPa) and a precise push-pull dynamometer (below 10KPa). The push-pull dynamometer was 
mounted onto a horizontal tensile test platform (Supplementary Fig. 5) to produce shear force for charactering piezoresistive effects under the stimuli from horizontal directions.

\section{The FE modelling and optimization of the sensor structure}

393 The tactile sensors with different auxetic structures were designed and modelled in Creo Parametric (PTC 394 Inc. Boston, US). Two key geometric parameters including the re-entrant angle (from $45^{\circ}$ to $90^{\circ}$ with the 395 increment of $\left.1^{\circ}\right)$ and the $\mathrm{H} / \mathrm{L}$ (Fig2.b) ratio $(\mathrm{H} / \mathrm{L}=2.7,2.0,1.61 .31 .0)$ were optimized. 230 tactile sensors with different gematrical parameters were modelled for structural optimization (46 re-entrant angles together with $5 \mathrm{H} / \mathrm{L}$ ratio, resulting in $46 \times 5=230$ different auxetic structures). The compression test was constant. The goal of the optimization was to achieve the largest nominal sensitivity among the sensors with different auxetic structures. The material property for the sensors were defined as linear elastic with the Young's Modulus of 2.0MPa and Poisson's ratio of 0.3. A flat plate was modelled to compress the sensor to a specified displacement. The bottom surface of the sensor was fixed and the mesh size of

$4040.5 \mathrm{~mm}$ with tetrahedral element was used for the simulation. 'Hard contact' was assigned between the flat 405 plate and the sensor, the 'self-contact' was defined over all the external and internal surfaces of the sensor 406 to measure the self-contact area during the compression. The strain energy density was extracted on the 407 nodes of the surfaces defined under self-contact for deriving the nominal sensor sensitivity. In total 230 408 simulations with different auxetic structures were carried out for the optimization to find the largest nominal sensitivity under the same external stimuli. The simulation results were also validated against the experimental data, a good agreement was achieved between nominal and real sensitivity. Therefore, the

411 FE simulation provided reliable optimization results of the structural design.

412 
415 The sensor printed onto the lumber vertebra and the distal femur head, connected with a customized 416 circuit composed of two shift registers, two multiplexers and an Arduino Uno board for collecting the

417 analog output from the tactile sensing elements. The analog output was converted into digital signals and 418 visualized through the GUI programmed in Processing IDE (Processing.org). The femur, vertebra and 419 inter-vertebra disc were 3D printed with UV curable resin as the target surfaces for printing the sensor.

420 A tendon-driven biomimetic hand containing intact hand bone skeleton, interphalangeal ligaments, 421 tendon and skin was employed in this study to demonstrate the sensorimotor control with the 3D printed 422 tactile sensor. The skeleton of the hand was 3D printed with polylactic acid (PLA) and the soft-tissues 423 were modeled with silicone-rubber. The anthropomorphic size of the biomimetic hand was reconstructed 424 from a 23-year-old male subject. Five electric motors (Dynamixel MX-12W, Robotics Inc., US) were 425 used to drive the biomimetic hand, each motor is corresponded to a human forearm muscle associated 426 with the hand grasping. The sensor was 3D printed onto the distal phalange of the index, connected with 427 the same customized electric circuit to collect the analog output from the tactile sensing elements and 428 convert it to digital signals as the feedback for controlling the biomimetic hand. The in-vivo grasping 429 experiment was carried out and the muscle forces under reactive and active touch were derived based on 430 the electromyographic signals collected by Delsys system (Delsys Trigno, Delsys Inc., US). The motors 431 of the biomimetic hand were then modulated to obtain the similar pulling forces with human forearm 432 muscles. A python program was developed for processing the digital signal input from the Uno board 433 connected with the tactile sensor, applying displacement and torque control algorithm to the motors and 434 restoring biological sensorimotor control function on biomimetic hand. Tactile signals of the biomimetic 435 hand under sensorimotor control and active touch were obtained, enabling the human-like grasping 436 performance of next generation prosthetics.

\section{Data Availability}

438 The source data including Supplementary Figures 1-7 and Supplementary Movie 1-4 are provided with 439 this paper. The 3D printing process of the tactile sensor on a finger bone is presented in Movie 1. Movie 2 
440 shows the FE simulations for the optimization of the auxetic structure of the sensor. Movie 3 displays the

441 measurement of the intervertebral disc pressure and the mechanical load acting at the hip joint using the

442 3D printed sensor. Movie 4 presents the measurement of the finger contact pressure and sensorimotor

443 control of a biomimetic hand based on the tactile feedback of the 3D printed sensor. The 3D model and

444 2D drawings of the propeosed tactile sensor can be found on figshare

445 (https://figshare.com/articles/figure/3D Model and Drawings of the Tactile Sensor NatCom 2021/16

446 569696). Data supporting the findings of this manuscript are available from the corresponding author

447 upon reasonable request.

\section{Code Availability}

449 The $\mathrm{G}$ code for 3D printing the sensor and the Python code for performing sensorimotor control on

450 biomimetic hand are $\quad$ available $\quad$ on $\quad$ OSF

451 (https://osf.io/p2uyt/?view_only=d62014436e414ce69ab22f0a496033b3). 
463

464

465

466

467

468

469

470

471

472

473

474

475

476

477

478

479

480

481

482

483

484

485

486

487

488

489

490

491

492

493

494

495

496

497

498

499

500

501

502

503

504

505

506

Chen, X. et al. Flexible three-axial tactile sensors with microstructure-enhanced piezoelectric effect and specially-arranged piezoelectric arrays. Smart Materials and Structures 27, 025018 (2018).

2 Choi, E. et al. Highly sensitive tactile shear sensor using spatially digitized contact electrodes. Sensors 19, 1300 (2019).

3 Li, X. et al. Highly sensitive flexible tactile sensors based on microstructured multiwall carbon nanotube arrays. Scripta Materialia 129, 61-64 (2017).

4 Luo, N. et al. Hollow-structured graphene-silicone-composite-based piezoresistive sensors: Decoupled property tuning and bending reliability. Advanced materials 29, 1702675 (2017).

5 Wang, Z. et al. Full 3D printing of stretchable piezoresistive sensor with hierarchical porosity and multimodulus architecture. Advanced Functional Materials 29, 1807569 (2019).

6 Ha, M. et al. Bioinspired interlocked and hierarchical design of $\mathrm{ZnO}$ nanowire arrays for static and dynamic pressure-sensitive electronic skins. Advanced Functional Materials 25, 2841-2849 (2015).

7 Jung, M. et al. Transparent and flexible Mayan-pyramid-based pressure sensor using facile-transferred indium tin oxide for bimodal sensor applications. Scientific reports $\mathbf{9}$, 1-11 (2019).

8 Kwon, D. et al. Highly sensitive, flexible, and wearable pressure sensor based on a giant piezocapacitive effect of three-dimensional microporous elastomeric dielectric layer. $A C S$ applied materials \& interfaces 8, 16922-16931 (2016).

9 Lee, Y. et al. Flexible ferroelectric sensors with ultrahigh pressure sensitivity and linear response over exceptionally broad pressure range. ACS nano 12, 4045-4054 (2018).

10 Park, J., Kim, M., Lee, Y., Lee, H. S. \& Ko, H. Fingertip skin-inspired microstructured ferroelectric skins discriminate static/dynamic pressure and temperature stimuli. Science advances 1, e1500661 (2015).

11 Park, J. et al. Tactile-direction-sensitive and stretchable electronic skins based on humanskin-inspired interlocked microstructures. ACS nano 8, 12020-12029 (2014).

12 Hasan, S. A. U. et al. A sensitivity enhanced MWCNT/PDMS tactile sensor using micropillars and low energy Ar+ ion beam treatment. Sensors 16, 93 (2016).

13 Wang, Y., Zhu, L., Mei, D. \& Zhu, W. A highly flexible tactile sensor with an interlocked truncated sawtooth structure based on stretchable graphene/silver/silicone rubber composites. Journal of Materials Chemistry C 7, 8669-8679 (2019).

14 Makihata, M. et al. Design and fabrication technology of low profile tactile sensor with digital interface for whole body robot skin. Sensors 18, 2374 (2018).

15 Oh, H., Yi, G.-C., Yip, M. \& Dayeh, S. A. Scalable tactile sensor arrays on flexible substrates with high spatiotemporal resolution enabling slip and grip for closed-loop robotics. Science advances 6, eabd7795 (2020).

16 Pyo, S., Lee, J.-I., Kim, M.-O., Lee, H.-K. \& Kim, J. Polymer-based flexible and multidirectional tactile sensor with multiple $\mathrm{NiCr}$ piezoresistors. Micro and Nano Systems Letters 7, 1-8 (2019).

17 Zhu, B. et al. Microstructured graphene arrays for highly sensitive flexible tactile sensors. Small 10, 3625-3631 (2014). 
18 Sun, X. et al. Flexible tactile electronic skin sensor with 3D force detection based on porous CNTs/PDMS nanocomposites. Nano-Micro Letters 11, 57 (2019).

19 Kou, H. et al. Wireless wide-range pressure sensor based on graphene/PDMS sponge for tactile monitoring. Scientific reports 9, 1-7 (2019).

20 Boutry, C. M. et al. A hierarchically patterned, bioinspired e-skin able to detect the direction of applied pressure for robotics. Science Robotics 3 (2018).

21 Han, Z. et al. Fabrication of highly pressure-sensitive, hydrophobic, and flexible 3D carbon nanofiber networks by electrospinning for human physiological signal monitoring. Nanoscale 11, 5942-5950 (2019).

22 Pang, C. et al. A flexible and highly sensitive strain-gauge sensor using reversible interlocking of nanofibres. Nature materials 11, 795-801 (2012).

23 Camilli, L. \& Passacantando, M. Advances on sensors based on carbon nanotubes. Chemosensors 6, 62 (2018).

24 Ma, P.-C. et al. Enhanced electrical conductivity of nanocomposites containing hybrid fillers of carbon nanotubes and carbon black. ACS applied materials \& interfaces $\mathbf{1}$, 1090-1096 (2009).

25 Obitayo, W. \& Liu, T. A review: Carbon nanotube-based piezoresistive strain sensors. Journal of Sensors 2012 (2012).

26 Yi, Z., Zhang, Y. \& Peters, J. Biomimetic tactile sensors and signal processing with spike trains: A review. Sensors and Actuators A: Physical 269, 41-52 (2018).

27 Koç, İ. M. \& Akça, E. Design of a piezoelectric based tactile sensor with bio-inspired micro/nano-pillars. Tribology International 59, 321-331 (2013).

28 Devaraj, H. et al. Bio-inspired flow sensor from printed PEDOT: PSS micro-hairs. Bioinspiration \& biomimetics 10, 016017 (2015).

29 Wan, Y. et al. A Highly Sensitive Flexible Capacitive Tactile Sensor with Sparse and High-Aspect-Ratio Microstructures. Advanced Electronic Materials 4, 1700586 (2018).

30 Amoli, V. et al. Biomimetics for high-performance flexible tactile sensors and advanced artificial sensory systems. Journal of Materials Chemistry C 7, 14816-14844 (2019).

31 Johnson, K. O. The roles and functions of cutaneous mechanoreceptors. Current opinion in neurobiology 11, 455-461 (2001).

32 Evans, K. E., Nkansah, M., Hutchinson, I. \& Rogers, S. Molecular network design. Nature 353, 124-124 (1991).

33 Kanda, Y. Piezoresistance effect of silicon. Sensors and Actuators A: Physical 28, 83-91 (1991).

34 Matsuda, K., Suzuki, K., Yamamura, K. \& Kanda, Y. Nonlinear piezoresistance effects in silicon. Journal of applied physics 73, 1838-1847 (1993).

35 Toriyama, T., Tanimoto, Y. \& Sugiyama, S. Single crystal silicon nano-wire piezoresistors for mechanical sensors. Journal of microelectromechanical systems 11, 605-611 (2002).

$36 \mathrm{Hu}, \mathrm{F}$. et al. Gel-Based Artificial Photonic Skin to Sense a Gentle Touch by Reflection. ACS applied materials \& interfaces 11, 15195-15200 (2019).

$37 \mathrm{Ji}, \mathrm{Z}$. et al. The design and characterization of a flexible tactile sensing array for robot skin. Sensors 16, 2001 (2016).

38 Kang, K., Cho, Y. \& Yu, K. J. Novel nano-materials and nano-fabrication techniques for flexible electronic systems. Micromachines 9, 263 (2018). 
55239 Tiwana, M. I., Redmond, S. J. \& Lovell, N. H. A review of tactile sensing technologies $553 \quad$ with applications in biomedical engineering. Sensors and Actuators A: physical 179, 1731 (2012).

40 Stassi, S., Cauda, V., Canavese, G. \& Pirri, C. F. Flexible tactile sensing based on piezoresistive composites: A review. Sensors 14, 5296-5332 (2014).

\section{Acknowledgements}

559 We would like to thank our research group members in University of Manchester and Jilin University for

560 their great support and assistant to this study. Funding: This research was partly supported by the project

561 of National Key R\&D Program of China (No. 2018YFC2001300), the project of National Natural Science

562 Foundation of China (No. 91948302, No. 91848204, No. 52005209, and No. 51675222). Competing

563 interests: The authors declare that they have no competing interests. 


\section{Supplementary Files}

This is a list of supplementary files associated with this preprint. Click to download.

- YuyangSupplementary.pdf

- Movie1.mp4

- Movie2.mp4

- Movie3.mp4

- Movie4.mp4 